\title{
Acute generalized exanthematous pustulosis induced by ceftriaxone use
}

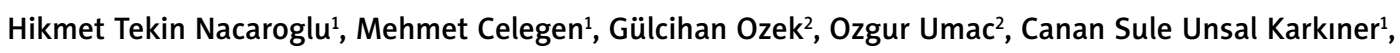 \\ Hulya Tosun Yıldırım ${ }^{3}$, Demet Can $^{1}$
}

\author{
'Department of Pediatric Allergy, Dr Behcet Uz Children's Hospital, Izmir, Turkey \\ Head of Department: Demet Can Assoc. Prof., MD \\ 2Department of Pediatric Hematology, Dr Behcet Uz Children's Hospital, Izmir, Turkey \\ Head of Department: Canan Vergin Assoc. Prof., MD \\ 3Department of Pathology, Dr Behcet Uz Children's Hospital, Izmir, Turkey \\ Head of Department: Gulden Diniz Assoc. Prof., MD
}

\begin{abstract}
Acute generalized exanthematous pustulosis (AGEP) is a rare cutaneous rash characterized by the abrupt onset of a generalized pustular rash often accompanied by fever. There is a history of drug use in $90 \%$ of the cases. Here we have reported a 15-year-old male patient with sickle cell anemia who developed AGEP after the use of ceftriaxone. Our patient was hospitalized because of vaso-occlusive crisis and on the third day of ceftriaxone treatment, erythematous pustular lesions accompanied with fever were observed on the body and extremities. Resolution of symptoms followed discontinuation of ceftriaxone. Sensitivity to ceftriaxone was shown with a patch test. The AGEP was considered due to clinical and histopathological findings. This is the first pediatric case of AGEP due to ceftriaxone.
\end{abstract}

Key words: acute generalized exanthematous pustulosis, children, drug hypersensitivity.

\section{Introduction}

Acute generalized exanthematous pustulosis (AGEP) is a rare cutaneous reaction which progresses with multiple pustular lesions on erythematous and edematous surface with a systemic fever [1]. It could be differentiated from other pustular eruptions like pustular psoriasis using the scoring system taken from the European Study of Severe Cutaneous Adverse Reactions (Euro SCAR) study. Although drugs, particularly antibiotics are reported to be major etiological factors, viral and bacterial infections and hypersensitivity reactions to mercury have also been accused [1-4]. In our study, an AGEP case due to ceftriaxone was presented.

\section{Case report}

A 15-year-old male patient who had been suffering from sickle cell anemia was admitted to our hospital because of chest pain and he was hospitalized at the Department of Hematology due to the pre-diagnosis of vaso-occlusive crisis and a consultation was requested from the Department of Pediatric Allergy because of a rash on his $3^{\text {rd }}$ day of hospitalization. There was no history of drug allergy and dermatosis. His weight was $56 \mathrm{~kg}(25-50 \mathrm{p})$, and his height was $166 \mathrm{~cm}$ (25-50 p). Physical examination revealed nothing abnormal in any of the other systems. In laboratory analyses, the following were found: hemoglobin: $7.9 \mathrm{~g} / \mathrm{dl}$, hematocrit: $25 \%$, white blood cell count: $10,600 / \mathrm{mm}^{3}$ (in peripheral blood smear, 46\% neutrophils, 50\% lymphocytes, 4\% monocytes), thrombocyte: $449,000 / \mathrm{mm}^{3}$, erythrocyte sedimentation rate: $15 \mathrm{~mm} / \mathrm{h}, \mathrm{C}$-reactive protein (CRP): 0.33 . The patient was put on ceftriaxone treatment for vaso-occlusive crisis and on the third day of the treatment, fever and numerous pustular lesions were seen on erythematous skin of his body and extremities (Figure 1). Mucosal involvement was not determined. He had leukocytosis $\left(17,400 \mathrm{~mm}^{3}\right)$ and CRP was positive $(8.74 \mathrm{mg} /$ dl). Punch biopsy from pustules revealed subcorneal pustular formation, perivascular infiltration rich in leukocyte

Address for correspondence: Hikmet Tekin Nacaroglu, Department of Pediatric Allergy, Dr Behcet Uz Children's Hospital, Izmir, Turkey, phone: +90 23241163 19, fax: +90232 48923 15, e-mail: tekin212@gmail.com

Received: 1.08 .2013 , accepted: 20.10.2013. 


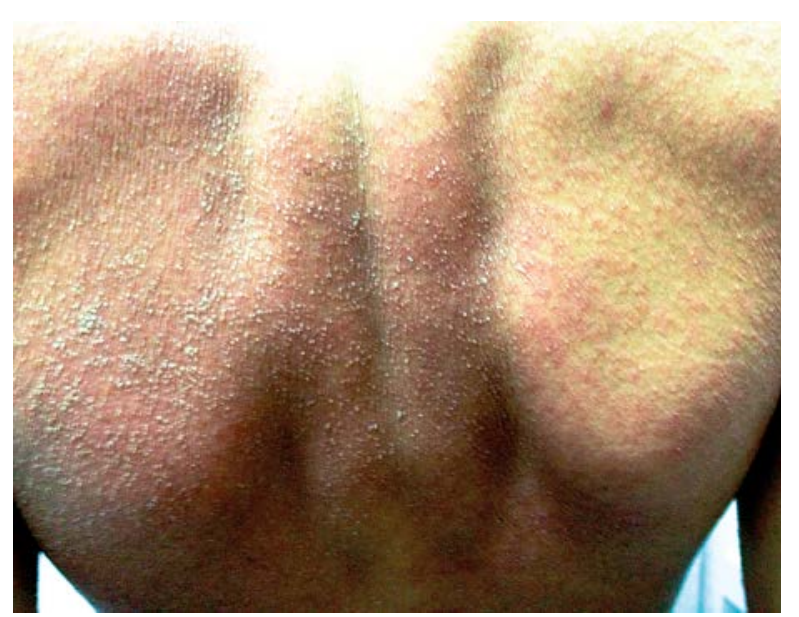

Figure 1. Pustular lesions on the erythematous surface of arms and the back

in dermis and acanthosis in epidermis. No accumulation was detected in immunofluorescence analyses. Clinical and histopathological findings were considered as AGEP. Ceftriaxone treatment was stopped, and antihistaminic one was commenced. The patient's rashes decreased within the following 3 days and then diminished with desquamation.

\section{Discussion}

The AGEP was first described by Beylot et al. [2] in 1980 , resembling very much to generalized pustular psoriasis but as a different pustular drug eruption which developed frequently due to medication (almost 90\%) [1-3]. Several drugs are reported to induce AGEP. Antibiotics (65\%), mainly macrolides (24\%) and $\beta$-lactamase (22\%) but also quinolones, tetracyclines, aminoglycosides, sulphonamides are the most common causative agents in AGEP. Other drugs that have also been implicated are calcium channel blockers, metronidazole, isoniazid, antimycotics, non-steroidal anti-inflammatory drugs (NSAIDs), paracetamol, furosemide, diltiazem, codeine, dexamethasone, carbamazepine and phenytoin [1-4].

The AGEP is a disease with acute onset having edematous and itchy features along with fever, swelling from erythematous surface, displaying integration and spreading on a large scale, nonfollicular, clinically characterized by innumerable < 5-mm-pustules' fast diffusion to torso within hours beginning from the face together with finger-gaps [4]. There can be an involvement of mucous membranes in $25 \%$ of cases [5]. Palms and soles are generally not involved. Pustules sometimes can unite and lead to pseudo-Nikolsky evidence $[1,5]$. Fever above $38^{\circ} \mathrm{C}$ is generally available and neutrophilia being over $7000 \mathrm{~mm}^{3}$ is seen in $80 \%$ of the patients. It is reported in one-third of the cases with subtle eosinophil [1, 4, 5]. Pustules spontaneously ameliorate with desquamations in a few

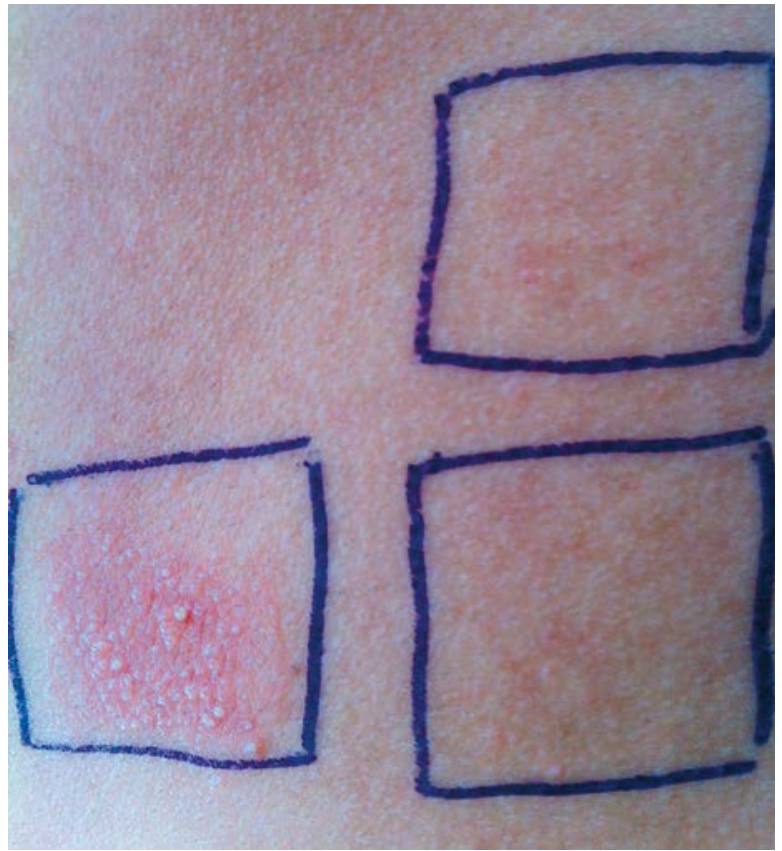

Figure 2. Patch test with $10 \%$ parenteral ceftriaxone was found to be positive

days. Reactions limit themselves in a period of less than fifteen days. Though internal organs are not usually involved, there can be a life-threatening condition as a result of secondary infections. The reported mortality rate is about 5\% [5]. Rashes had appeared on the face in the first place and then spread to the torso and extremities in our case. No mucosal involvement was determined. Following the cessation of drug intake, lesions regressed and recovered with desquamation. Clinical characteristics were among the findings verifying AGEP diagnosis such as fever commencing after drug intake and rapid spread of lesions along with recovery observed following giving up taking drugs.

The AGEP diagnosis can be made on the basis of the patient's history and clinical picture, and histopathological evidence supports its diagnosis. Intraepidermal subcorneal spongio form pustules are determined in histopathological analyses. Edema in papillary dermis, vasculutis, perivascular eosinophil infiltration and focal keratinous necrosis are other findings [4, 6-8]. Immunofluorescent studies are usually nonspecific [8]. In the skin biopsy evaluation carried out for our patient, parakeratotic type hyperkeratosis on the surface, acanthosis in the epidermis, pustule formation in a number of areas, necrotic keratinocytes as well as polymorphonuclear leukocytes infiltrating epidermis were seen. The AGEP diagnosis was established thanks to these findings.

A patch test is positive in many cases. Because of this, type IV hypersensitivity reaction was suspected to play a role in the pathogenesis [6-8]. Recent studies suggested interleukin-8 (IL-8) production which is neutro- 
phil-attracting and generated by T cell clones associated with drug in the skin and circulation were proved to play a role in AGEP [5, 7-10]. In our case, 10\% concentration of the commercial form of ceftriaxone parenteral and 0.9\% $\mathrm{NaCl}$ patch test was done. In the patch test area, pustular lesions on the erythematous surface was seen as reported elsewhere (Figure 2). The AGEP was reported in 2 patients aged 31 and 70 using ceftriaxone for acute pyelonephritis [11, 12]. No sensitivity level was shown with a patch test in these cases. Özmen et al. [13] reported AGEP following the use of ampicillin-sulbactam, clindamycin, amikacin, cefixime in 6-, 9-, 11- and 16-year-old patients and a patch test with ampicillin produced the positive result in one of the cases.

The AGEP diagnosis verification scoring formed by the Euro SCAR group, which is an international pharmacoepidemiological case-control study group can also be used [13-15]. A definitive diagnosis for AGEP was specified as a score ranging from 8 to 12 . Our case had a score of 12 according to this scoring system.

There is no treatment special to AGEP. Treatment is generally performed for symptoms. A spontaneous recovery is seen following the removal of the underlying cause of the discomfort. According to the prevalence of lesions and the general condition of the patient, systemic antihistaminics, topical moisturisers and lotions with corticosteroids are usually adequate in treatment [9-15]. In our case, topical moisturiser and antihistaminic treatments were applied after quitting drug therapy. The rashes of the disease regressed in 2-3 days and its lesions disappeared at the end of a week with desquamation.

\section{Conclusions}

The patient received the diagnosis as AGEP clinically and histopathologically and his symptoms regressed following the discontinuation of ceftriaxone which is thought to be the cause as the patient's ceftriaxone susceptibility (which was confirmed by a patch test). This is the first pediatric AGEP case related to ceftriaxone ever reported. As these reactions are known to be rare to emerge depending upon frequently used drugs, we have intended to draw attention to infections seen in childhood in the etiology of the rashes as well as drug reactions.

\section{Conflict of interest}

The authors have no conflicts of interest to disclose.

\section{References}

1. Chaabane A, Aouam K, Gassab L, et al. Acute generalized exanthematous pustulosis (AGEP) induced by cefotaxime. Fundam Clin Pharmacol 2010; 24: 429-32.
2. Beylot C, Bioulac P, Doutre MS. Acute generalized exanthematic pustuloses (four cases). Ann Dermatol Venereol 1980; 107: 37-48.

3. Freedberg IM, Eisen AZ, Wolff K, et al. Fitzpatrick's dermatology in general medicine. $6^{\text {th }}$ ed. McGraw-Hill, New York 2003; 625-27.

4. Roujeau JC, Bioulac-Sage P, Bourseau C, et al. Acute generalized exanthematous pustulosis. Arch Dermatol 1991; 127: 1333-8.

5. Halevy S, Kardaun SH, Davidovici B, et al.; EuroSCAR and RegiSCAR study group. The spectrum of histopathological features in acute generalized exanthematous pustulosis: a study of 102 cases. Br J Dermatol 2010; 163: 1245-52.

6. Wolkenstein $\mathrm{P}$, Chosidow O, Flechet ML, et al. Patch testing in severe cutaneous adverse drug reactions, including Stevens-Johnson syndrome and toxic epidermal necrolysis. Contact Dermatitis 1996; 35: 234-6.

7. Kardaun SH, Kuiper H, Fidler V, et al. The histopathological spectrum of acute generalized exanthematous pustulosis (AGEP) and its differentiation from generalized pustular psoriasis. J Cutan Pathol 2010; 37: 1220-9.

8. Vicente-Calleja JM, Aguirre A, Landa N, et al. Acute generalized exanthematous pustulosis due to diltiazem: confirmation by patch testing. Br J Dermatol 1997; 137: 837-9.

9. Watsky KL. Acute generalized exanthematous pustulosis induced by metronidazole: the role of patch testing. Arch Dermatol 1999; 135: 93-4.

10. Beylot C, Doutre MS, Beylot-Barry M. Acute generalized exanthematous pustulosis. Semin Cutan Med Surg 1996; 15: 244-9.

11. Belda Junior W, Ferolla AC. Acute generalized exanthematous pustulosis (AGEP). Case report. Rev Inst Med Trop Sao Paulo 2005; 47: 171-6.

12. Kaya A, Yıldız S, Balkan II, et al. Acute generalized exanthematous pustulosis after ceftriaxone use resembling sepsis. J Infect Chemother 2012; 18: 112-4.

13. Özmen S, Misirlioglu ED, Gurkan A, et al. Is acute generalized exanthematous pustulosis an uncommon condition inchildhood? Allergy 2010; 65: 1490-2.

14. Sidoroff A, Halevy S, Bavinck JN, et al. Acute generalized exanthematous pustulosis (AGEP - a clinical reaction pattern. J Cutan Pathol 2001; 28: 113-9.

15. Sidoroff A, Dunant A, Viboud C, et al. Risk factors for acute generalized exanthematous pustulosis (AGEP) results of a multinational case-control study (EuroSCAR). Br J Dermatol 2007; 157: 989-96 